\title{
Dudleya brittonii extract promotes survival rate and M2-like metabolic change in porcine 3D4/31 alveolar macrophages
}

\author{
Hyungkuen Kim', Eek Hyung Jeon ${ }^{2}$, Byung-Chul Park ${ }^{3, *}$, and Sung-Jo Kim ${ }^{1, *}$
}

\author{
* Corresponding Authors: \\ Byung-Chul Park \\ Tel: +82-33-339-5792, Fax: +82-33-339-5763 \\ E-mail: bcpark@snu.ac.kr \\ Sung-Jo Kim \\ Tel: +82-41-540-5571, Fax: +82 41-540-9538 \\ E-mail: sungjo@hoseo.edu
}

1 Division of Cosmetics and Biotechnology, College of Life and Health Sciences, Hoseo University, Baebang, Asan 31499, Korea

2 Department of Biotechnology and Bioinformatics,

College of Science and Technology, Korea University, Sejong 30019, Korea

${ }^{3}$ Graduate School of International Agricultural

Technology and Institute of Green-Bio Science and

Technology, Seoul National University, Pyeongchang

25354, Korea

ORCID

Hyungkuen Kim

https://orcid.org/0000-0001-7508-9933

Eek Hyung Jeon

https://orcid.org/0000-0002-5070-6066

Byung-Chul Park

https://orcid.org/0000-0003-1489-8678

sung-Jo Kim

https://orcid.org/0000-0003-4590-3644

Submitted Mar 27, 2019; Revised Apr 5, 2019; Accepted Apr 11, 2019
Objective: Although alveolar macrophages play a key role in the respiratory immunity of livestock, studies on the mechanism of differentiation and survival of alveolar macrophages are lacking. Therefore, we undertook to investigate changes in the lipid metabolism and survival rate, using 3D4/31 macrophages and Dudleya brittonii which has been used as a traditional asthma treatment.

Methods: 3D4/31 macrophages were used as the in vitro porcine alveolar macrophages model. The cells were activated by exposure to phorbol 12-myristate 13-acetate (PMA). Dudleya brittonii extraction was performed with distilled water. For evaluating the cell survival rate, we performed the water-soluble tetrazolium salt cell viability assay and growth curve analysis. To confirm cell death, cell cycle and intracellular reactive oxygen species (ROS) levels were measured using flow cytometric analysis by applying fluorescence dye dichlorofluorescein diacetate and propidium iodide. Furthermore, we also evaluated cellular lipid accumulation with oil red $\mathrm{O}$ staining, and fatty acid synthesis related genes expression levels using quantitative polymerase chain reaction ( $\mathrm{qPCR}$ ) with SYBR green dye. Glycolysis, fatty acid oxidation, and tricarboxylic acid (TCA) cycle related gene expression levels were measured using qPCR after exposure to Dudleya brittonii extract (DB) for $12 \mathrm{~h}$.

Results: The ROS production and cell death were induced by PMA treatment, and exposure to $\mathrm{DB}$ reduced the PMA induced downregulation of cell survival. The PMA and DB treatments upregulated the lipid accumulation, with corresponding increase in the acetyl-CoA carboxylase alpha, fatty acid synthase mRNA expressions. DB-PMA co-treatment reduced the glycolysis genes expression, but increased the expressions of fatty acid oxidation and TCA cycle genes.

Conclusion: This study provides new insights and directions for further research relating to the immunity of porcine respiratory system, by employing a model based on alveolar macrophages and natural materials.

Keywords: Porcine; Alveolar Macrophage; Dudleya brittonii; Reactive Oxygen Species; Fatty Acid; Immunity

\section{INTRODUCTION}

Worldwide rapid climate changes (heat wave, fine dust, etc.) and high rates of dense breeding have negatively affected livestock immunity [1-3]. This trend has been exacerbated by the prohibition of growth-promoting hormones and antibiotics in livestock breeding [4]. Compared to cancer or dementia, respiratory infectious diseases that spread easily are of graver concern for farmhouses. This has resulted in increased importance focused on livestock health and respiratory immunity [5].

In general, blood circulating monocytes $(\mathrm{CMs})$ are derived from bone marrow, while alveolar macrophages (AMs) are specific tissue macrophages which form a few days after birth; briefly, fetal monocytes differentiate into AMs and settle in the alveolar tissue [6]. In 
addition, AMs encompass a self-renewal ability, which maintains the characteristics of existing AMs and embraces alveolar characteristics [7]. The CMs from bone marrow helps restore the number of AMs when the population decreases due to severe infection or radiotherapy, etc. [8]. The specific characteristics of AMs implies that CM-based studies are not applicable to AMs. Hence, using AMs such as 3D4/31, are more appropriate to study pulmonary health and immunity.

Previously, classical M1 macrophages were closely associated with the glycolysis pathway, which is a key regulator of macrophage activation. However, recent studies on M2-like macrophages such as AMs report that lipid metabolism plays an important role in macrophage differentiation and activation of the M2 form. Two basic lipid metabolisms (fatty acid synthesis [FAS] and fatty acid oxidation [FAO]) are known to work centrally [9]. The FAS pathway can be summarized as follows: carboxylation of acetyl-CoA to malonyl-CoA by acetyl-CoA carboxylase alpha (ACACA), and the production of palmitic acid (most basic fatty acids found in mammals) from malonyl-CoA or acetyl-CoA by fatty acid synthase (FASN) [10]. Using these or externally absorbed lipids, adenosine triphosphate (ATP) is produced through the FAO and tricarboxylic acid (TCA) cycle. The FAO pathway functions by converting fatty-acyl-CoA to acyl-CoA by the action of carnitine palmitoyltransferase (CPT), subsequent to betaoxidation in the mitochondrial matrix by several enzymes include acetyl-CoA acyltransferase (ACAA), and finally production of acetyl-CoA by $\beta$-oxidation, and involvement in the TCA-cycle for generating ATP [11].

Macrophage also have signaling to regulates excessive lipid accumulation. Enhanced steroid receptor coactivator-3 (SRC3) signaling promotes the metastasis of lung cancer and myeloma cells, which are known to be fatal in patients $[12,13]$. However, SRC3 plays an essential role in lipid metabolism and macrophage survival, by initiating FAO and preventing excessive fatty acid accumulation by reacting sensitively to fatty acid concentration [14]. Moreover, SRC3 signaling is activated by infection, thereby increasing the expression of antioxidant enzymes (catalase) and anti-apoptotic B-cell lymphoma 2 to reduce reactive oxygen species (ROS) generation and cell death, and improve bacterial clearance [15]. However, this insight into the AMs has not yet been achieved.

Plants are one of the most remarkable materials for enhancing livestock immunity and replacing antibiotics with low toxicity and various functionalities [16]. Since the research on the medical efficacy of plants has been continuously carried out and most of the plants have been studied, there is a growing demand for new plant species that have not been studied previously. The succulent plant, represented by aloe, is inhabited in unique environments such as desert, tropical regions, and coasts with high salinity, and has undergone relatively less research than other plant species [17]. Never- theless, a small number of succulent plants, such as aloe and prickly pear, have been successfully used for commercial purposes because of their proven efficacy in immunity $[18,19]$. For this study, we selected Dudleya brittonii (D. brittonii) from several succulent plant species by collecting various information about respiratory immunity. D. brittonii, also called giant chalk dudleya, is a succulent plant mainly found in California and Mexico, and is widely used for ornamental purposes [20]. Due to a dearth of biological studies on $D$. brittonii, the effect on cells or animals is yet to be elucidated. Interestingly, according to the University of San Diego, Kumeyaay, a native American in California, used D. brittonii for treating asthma, an inflammatory airway obstruction disease closely associated with macrophages. Therefore, in this study, we attempt to verify the survival and metabolic efficacy of D. brittonii extract (DB) in AMs.

\section{MATERIALS AND METHODS}

\section{Preparation of Dudleya brittonii extract}

Thirty grams of $D$. brittonii leaves were mixed with $300 \mathrm{~mL}$ of distilled water (DW), and extraction was performed at $110^{\circ} \mathrm{C}$ and $39.23 \mathrm{kPa}$ for $15 \mathrm{~min}$ in an autoclave (Daihan Scientific, Wonju, Korea). The aqueous phase was collected and filtered with a $0.2 \mu \mathrm{m}$ syringe filter (Minisart, Sartorius, Goettingen, Germany).

\section{Cell culture}

3D4/31 macrophages (ATCC-CRL-2844; ATCC, Manassas, VA, USA) were cultured in complete Roswell Park Memorial Institute (RPMI) 1640 medium (supplemented with 10\% [v/ v] fetal bovine serum and $1 \%[\mathrm{v} / \mathrm{v}]$ penicillin streptomycin) in a $\mathrm{CO}_{2}$ incubator $\left(95 \%\right.$ air and $\left.5 \% \mathrm{CO}_{2}, 37^{\circ} \mathrm{C}\right)$.

\section{Cell survival rate analysis}

To measure the survival rate, water-soluble tetrazolium salt (WST) - 1 cell viability assay and growth curve analysis were performed, as described previously [21]. Briefly, $3 \times 10^{3} 3 \mathrm{D} 4 / 31$ macrophages were seeded in 96-well cell culture plates (SPL Life Sciences, Pocheon, Korea); after 24 h, cells were treated with DB $(900,9,0.09 \mathrm{ng} / \mathrm{mL})$ and/or $2 \mathrm{nM}$ phorbol 12-myristate 13-acetate (PMA; Sigma-Aldrich, St. Louis, MO, USA), in triplicates. To determine cell viability, WST-1 reagent (EZ Cytox Cell Viability Assay Kit; DoGenBio, Seoul, Korea) was added to each well, and absorbance was measured at $450 \mathrm{~nm}$ using a microplate reader (Sunrise, Tecan, Männedorf, Switzerland). Each absorbance was compared to the vehicle control. For growth curve analysis, $5 \times 10^{4}$ cells were seeded in $60-\mathrm{mm}$ cell culture dishes (SPL Life Sciences, Korea) and incubated for $24 \mathrm{~h}$. Cells were then exposed to DB ( $9 \mathrm{ng} / \mathrm{mL}$ ), with/ without $2 \mathrm{nM}$ PMA, and fresh media was replenished every $24 \mathrm{~h}$. Cell numbers were assessed every $24 \mathrm{~h}$ using a hemocyto- 
meter and 0.4\% (w/v) trypan blue (Thermo Fisher Scientific, Waltham, MA, USA) staining. Microscopic analysis was performed using the CKX41 microscope (Olympus Optical Co., Center Valley, PA, USA) and AxioCam HRc (Carl Zeiss, Oberkochen, Germany). Images were processed with the Axio vision Rel. 4.7 (Carl Zeiss) and Photoshop CC 2018 program (Adobe Systems, San Jose, CA, USA).

\section{Oxidative stress analysis}

Intracellular ROS levels of 3D4/31 macrophages were measured using the ROS indicator dichlorofluorescein diacetate (DCFDA, Sigma-Aldrich, USA), as described before [21]. Briefly, $5 \times 10^{5}$ cells were seeded in a $60-\mathrm{mm}$ cell culture dish. After $12 \mathrm{~h}$, cells were exposed to DB $(900,9,0.09 \mathrm{ng} / \mathrm{mL})$ with/without $2 \mathrm{nM}$ PMA, using DW as vehicle, followed by $12 \mathrm{~h}$ incubation. DCFDA (final concentration of $10 \mu \mathrm{M}$ ) was administered for $30 \mathrm{~min}$, after which the cells were harvested and washed with phosphate buffered saline (PBS; pH 7.4 ). DCF-median fluorescence intensity was detected by the Guava EasyCyte (Millipore, Temecula, CA, USA) and FlowJo (TreeStar, Ashland, OR, USA), and relative ROS levels were measured as compared to vehicle.

\section{Cell cycle analysis}

Propidium iodide (PI; Sigma-Aldrich, USA) was used for cell cycle analysis, as described before [22]. Briefly, $5 \times 10^{5} 3 \mathrm{D} 4 / 31$ macrophages were seeded in a $60-\mathrm{mm}$ cell culture dish and incubated for $12 \mathrm{~h}$. The cells were treated with DB $(9 \mathrm{ng} / \mathrm{mL})$ with/without $2 \mathrm{nM}$ PMA for $12 \mathrm{~h}$. Treated cells were then fixed and permeabilized using ice-cold ethanol at $-20^{\circ} \mathrm{C}$, washed, and resuspended in PBS, followed by addition of RNase A (final concentration at $250 \mu \mathrm{g} / \mathrm{mL}$ ). After $30 \mathrm{~min}$ incubation room temperature $\left(22^{\circ} \mathrm{C}\right)$, cells were stained with PI solution (final concentration at $500 \mu \mathrm{g} / \mathrm{mL}$ ) for $15 \mathrm{~min}$ in the dark at $4^{\circ} \mathrm{C}$, PI fluorescence intensity was measured using Guava EasyCyte and FlowJo.

\section{Lipid staining and concentration measurements}

Oil red $\mathrm{O}$ was used for lipid staining, as described before, with minor modification [23]. Briefly, $5 \times 10^{5} 3 \mathrm{D} 4 / 31$ macrophages were seeded in a $60-\mathrm{mm}$ cell culture dish. After 12 $\mathrm{h}$, cells were treated with $\mathrm{DB}(9 \mathrm{ng} / \mathrm{mL})$ and/or $2 \mathrm{nM}$ PMA for $24 \mathrm{~h}$. The cells were washed with PBS and fixed in 3.8\% formaldehyde for $15 \mathrm{~min}$, washed with 60\% (v/v) isopropanol, air dried, and stained with oil red O (Sigma Aldrich, USA; $0.2 \mu \mathrm{m}$ filtered, $0.3 \%[\mathrm{w} / \mathrm{v}$ ] oil red $\mathrm{O}$ in $60 \%[\mathrm{v} / \mathrm{v}]$ isopropanol). Stained cells were then washed with DW and examined for oil red $\mathrm{O}$ staining using the DMi8 fluorescence microscope (Leica, Deerfield, IL, USA). Images were processed by applying LAS X (Leica, USA) and Photoshop CC 2018 program.
Quantitative polymerase chain reaction

Briefly, $5 \times 10^{5}$ cells $3 \mathrm{D} 4 / 31$ macrophages were seeded in a $60-\mathrm{mm}$ cell culture dish for RNA isolation. After $24 \mathrm{~h}$, cells were exposed to DB ( $9 \mathrm{ng} / \mathrm{mL}$ ) with/without $2 \mathrm{nM}$ PMA, and incubated for further $12 \mathrm{~h}$. Total RNA was extracted using TRIzol (Thermo Fisher Scientific, USA), according to the manufacturer's manual. cDNA synthesis of 3D4/31 macrophages was performed using the MLV-RT cDNA synthesis kit (Wizbiosolutions, Seongnam, Korea) as per the manufacture's manual. Total $10 \mathrm{ng}$ of cDNA was used for quantitative polymerase chain reaction (qPCR) using the $2 X$ SYBR Green PCR Master Mix (Biofact, Daejeon, Korea), performed according to the manufacturer's instructions. Specific primer sequences are described in Table 1 . The qPCR was performed by using the StepOnePlus Real-Time PCR System (Applied Biosystems, Foster City, CA, USA), and mRNA fold change was analyzed using the $2^{-\Delta \Delta C \mathrm{t}}$ method [24].

\section{Statistical analysis}

All experiments were repeated at least three times and data were obtained from three separate experiments. All data are expressed as the mean \pm standard deviation (SD). Analysis was performed by GraphPad PRISM 8 (GraphPad Software, San Diego, CA, USA) and Microsoft Excel (Office 365; Microsoft, Redmond, WA, USA). Two-tailed Student's $t$-test was used to measuring the p-value. Data are considered statistically significant when the $\mathrm{p}$-value is $<0.05$.

\section{RESULTS}

\section{Dudleya brittonii extract protect 3D4/31 macrophages against phorbol phorbol 12-myristate 13-acetate induced cell death}

To test the protective effect of DB on decreasing cell viability in activated macrophages, we performed the WST-1 assay after exposing cells to DB or PMA for $24 \mathrm{~h}$. As compared to vehicle control, we observed a dose-dependent decrease in viability after exposure to $\mathrm{DB}$, and viability was decreased by $14.77 \% \pm 4.2 \%$ after PMA treatment. However, co-treatment with DB and PMA for $24 \mathrm{~h}$ significantly increased the cell viability by up to $11.87 \% \pm 4.62 \%$ as compared to PMA treated cells (Figure 1A; $\mathrm{p}<0.01$ ). The WST-1 cell viability assay showed a dose-dependent viability-enhancing effect of $\mathrm{DB}$ on activated 3D4/31 macrophages, and confirmed the highest activity at $9 \mathrm{ng} / \mathrm{mL}$ concentration. Therefore, we performed the growth curve analysis at a final concentration of $9 \mathrm{ng} / \mathrm{mL}$ to confirm the effect of $\mathrm{DB}$ on the proliferation rate of 3D4/31 macrophages. In this assay, cells were exposed to DB with/without PMA every $24 \mathrm{~h}$. We observed that PMA decreases the cell number by $44.14 \% \pm 6.66 \%$ as compared to vehicle, but co-treatment with DB increases the cell number by $45.13 \% \pm 16.81 \%$ as compared to PMA treated cells 
Table 1. Primer sequences for fatty acid synthesis, fatty acid oxidation, glycolysis, and tricarboxylic acid cycle

\begin{tabular}{|c|c|}
\hline Target genes & Sequences $\left(5^{\prime}\right.$ to $\left.3^{\prime}\right)$ \\
\hline FASN & F: GTGGAGGTGCGCCAGATACT \\
\hline (Fatty acid synthase) & R: CCTCGTGGGATGTGGGAGTC \\
\hline$A C A C A$ & F:ACAGCTGACGGAGGAAGACG \\
\hline (Acetyl-CoA carboxylase alpha) & R: GCTCGCTGAGTGGGTGAGAT \\
\hline SRC3 & F:TCAAAGGCCAGCCGAATGGA \\
\hline (Steroid receptor coactivator-3) & R: CCAGGCCGCATTTGAAGCAT \\
\hline SRA1 & F:AGCTTCCAGGCCTCCACTTG \\
\hline (Steroid receptor RNA activator 1) & R: GCCAGGCGTCGGTTTATGTC \\
\hline GLUT1 & F: CTGCTCATCAACCGCAATGA \\
\hline (Glucose transporter 1) & R: GGCTCTCCTCCTTCATCTCC \\
\hline HK2 & F: CACTGCTGAAGGAAGCCATC \\
\hline (Hexokinase2) & R: GGGTCTTCATAGCCACAGGT \\
\hline HK3 & F: CTCTGGAGGTGTGCAGATCA \\
\hline (Hexokinase3) & R: TTCTGCTGGAAGTCCACGAT \\
\hline PFKP & F: CCGACGGACACAAGATGTTC \\
\hline (Phosphofructokinase platelet) & R:TTGTCCCAAGAATGGAGCCT \\
\hline PFKL & F: GAAACGAGAAGTGCCACGAA \\
\hline (Phosphofructokinase liver) & R:TACCGTAGTTCCGGTCGAAG \\
\hline$P K L R$ & F:AGACTGCCAAGGGTCACTTT \\
\hline (Pyruvate kinase liver \& red blood cells) & R: CAGCTCCTCAAAGAGTTGCC \\
\hline PKM & F: GATCCCAAGACTCTGGCCTT \\
\hline (Pyruvate kinase muscle) & R: CCAGCTTGTCCATCTGCTTC \\
\hline LDHAL6B & F: GATTGGGCAGAGGCTTGGTA \\
\hline (L-lactate dehydrogenase A-like 6B-like isoform) & R: CACCAGCGATGTTCACTCCA \\
\hline LDHA & F: TAATGGGGGAAAGGCTGGGA \\
\hline (Lactate dehydrogenase A) & R: CGCTCCATACAGGCACACTA \\
\hline CPT1A & F: CAAGATAGCGGCCGAAAAGC \\
\hline (Carnitine palmitoyltransferase 1a) & R: GATAATCGCCACGGCTCAGA \\
\hline CPT1B & F: CCACTATGACCCGGAAGACG \\
\hline (Carnitine palmitoyltransferase 1b) & R: TTGAACGCGATGAGGGTGAA \\
\hline$A C A D V L$ & F: GCGGTGAATCATGCTGCTAA \\
\hline (Very long-chain acyl-CoA dehydrogenase) & R: GTGGATCCCTGGTCCATGTT \\
\hline$A C A D L$ & F: GCGTGGCTTATGACTGTGTG \\
\hline (Long-chain acyl-CoA dehydrogenase) & R:ACTCGGGCATCCACATAAGC \\
\hline$A C A A 1$ & F:TCGCCCAGTTTCTGAGTGAC \\
\hline (Acetyl-CoA acyltransferase 1A) & R: CCACAAGCCATGCCAATGTC \\
\hline ACAA2 & F:TCGTGGGCTATTTTGCGTCT \\
\hline (Acetyl-CoA acyltransferase 2) & R:TCCTGCTTTCTTCAGTGCCC \\
\hline PPARA & F:TTGAACGACCAGGTCACGCT \\
\hline (Peroxisome proliferator-activated receptor $\alpha$ ) & R: GGAACTCGCGCGTGATGAAG \\
\hline DLAT & F:AGCTTCAGCCTTGGCATGTT \\
\hline (Dihydrolipoamide S-acetyltransferase) & R:TGCTGACTGCGACACTGATA \\
\hline CTS & F: GGGCACTGGGTGTATTAGCA \\
\hline (Citrate synthase) & R:TCATGGACTTGGGCCTCTCT \\
\hline IDH3 & F: CGCTGCAAAGATTGAGACCG \\
\hline (Isocitrate dehydrogenase 3 alpha) & R:TCTGAGCATTTTGCGTTGCC \\
\hline SUCLG1 & F: CAGGGCACCTTTCATAGCCA \\
\hline (Succinate thiokinase) & R: CCTTTCCCTGGAGTGGTTCC \\
\hline $\mathrm{MDH} 1$ & F:TGGTGTTCCTGATGATCTGCTC \\
\hline (Malate dehydrogenase 1) & R: CCTTTGCAGTGAGGTCCATCTT \\
\hline $\mathrm{MDH} 2$ & F: CAACCCGGTTAACTCCACCA \\
\hline (Malate dehydrogenase2) & R:ACCCTTCAGCTCTGCAACAA \\
\hline GAPDH & F: GTCGGAGTGAACGGATTTGGC \\
\hline (Glyceraldehyde 3-phosphate dehydrogenase) & R:ACTGTGCCGTGGAATTTGCC \\
\hline
\end{tabular}

F: forward; R: reverse.
(Figure 1B; $\mathrm{p}<0.05$ ). In addition, the phenotype was observed to confirm for the inhibitory effect of DB on 3D4/31 macrophages activation. No specific phenotypic difference was observed between the PMA-treated and PMA-DB cotreated groups (Figure 1C).

Our results indicate that the survival rate of 3D4/31 macrophages was dramatically decreased when activated by PMA, and DB effectively prevents the PMA-induced inhibition of survival rate. Unlike M1 macrophages (which are mainly involved in phagocytosis), AMs (M2 type) survive longer and are important for wound healing and anti-inflammation [25]. Thus, we confirmed that the survival protection efficacy of $\mathrm{DB}$ is associated with respiratory immunity controlled by AMs.

\section{Dudleya brittonii extract exerts anticytotoxic and antioxidant effect in phorbol 12-myristate 13-acetate treated cells}

Inhibition of cell survival rate by macrophage activation results from excessive changes in the cell cycle and cell death due to excessive ROS production. We therefore examined the effect of DB on the cell cycle of macrophages treated with/ without PMA; cell cycle and cell death were evaluated by PI staining and flow-cytometric analysis. We observed that DB does not induce any significant change in the cell cycle either by applying single treatment or co-treatment with PMA, in $3 \mathrm{D} 4 / 31$ macrophages. However, the dead cells population was increased 2-fold as compared to the vehicle when DB was cotreated with PMA; furthermore, exposure to DB also reduces the number of dead cells as compared to the vehicle only treated cells (Figure 2A, 2B; $\mathrm{p}<0.05$ ). These results demonstrate that $\mathrm{DB}$ reduces the cell death in activated macrophages, and provides evidence of enhanced cell survival effect after DB exposure. We simultaneously confirmed the effects of $\mathrm{DB}$ on ROS production (12 h after treatment with $\mathrm{DB}$ and/ or PMA). DCFDA, a plasma membrane permeable molecule and sensitive indicator of ROS, was applied for the investigation. We observed intracellular reduction of ROS levels $(20.93 \% \pm 2.89 \%)$ in $3 \mathrm{D} 4 / 31$ macrophages after treatment with $\mathrm{DB}$, as compared to vehicle treatment and PMA treatment $(25.08 \% \pm 12.41 \%$ increase after PMA treatment). Furthermore, DB-PMA co-treatment reduced the ROS level by $23.43 \% \pm$ $8.54 \%$ as compared to PMA treatment (Figure 2C, 2D; $\mathrm{p}<$ 0.05). Taken together, these results indicate that the PMA induced 3D4/31 macrophage activation induces ROS production, and DB has an antioxidant effect on both non-activated and PMA-activated 3D4/31 macrophages.

Activated M1 macrophage ROS plays an important role in phagocytosis, but excessive ROS production induces inflammation and apoptosis by activating pro-inflammatory signaling such as tumor necrosis factor- $\alpha$ and nuclear factor $\kappa$-light-chain-enhancer of activated B cells [26]. However, M2-like AMs do not require excessive ROS production to 


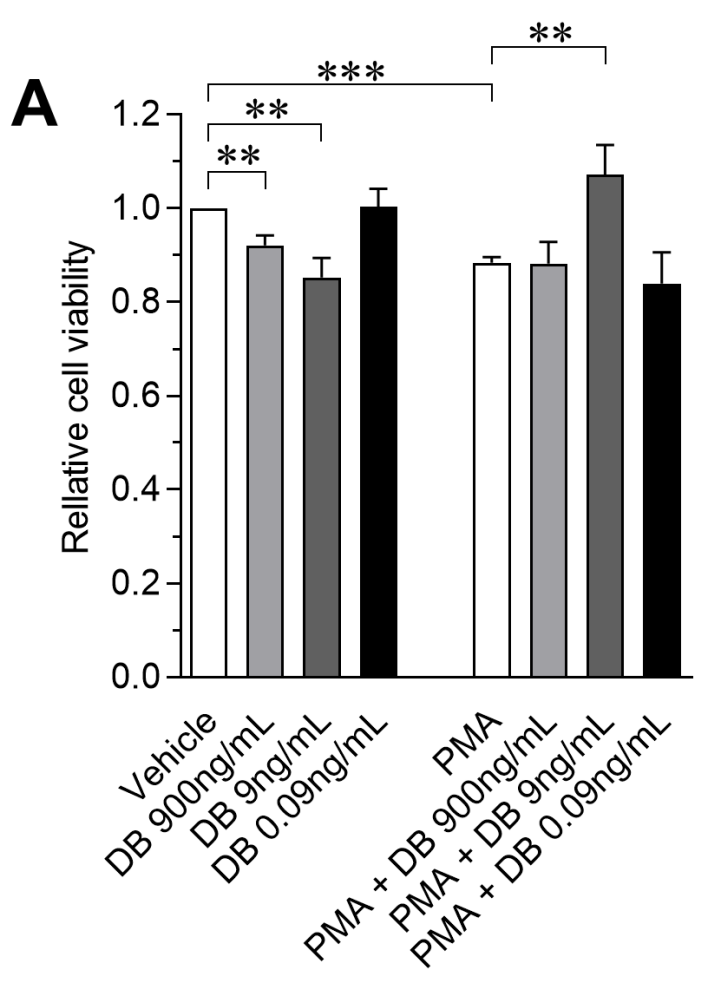

C

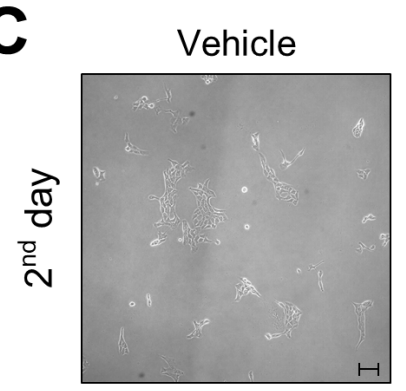

DB

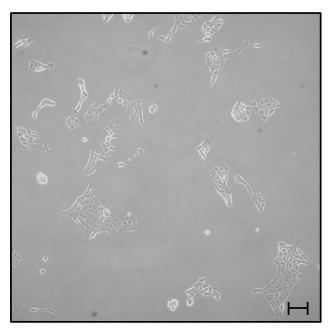

B

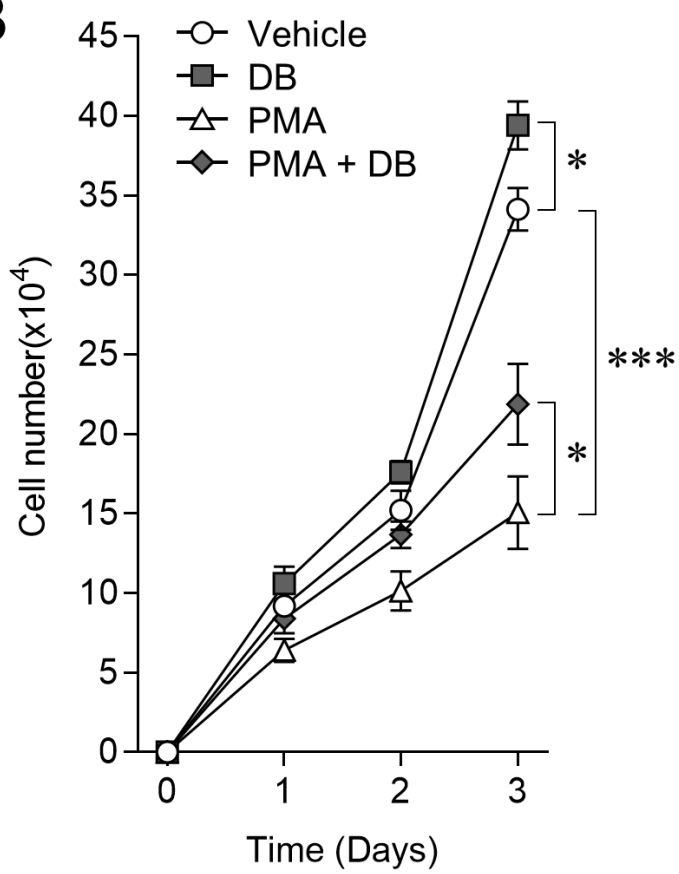

Figure 1. Inhibitory effect of DB on decreased PMA-induced cell survival rate in 3D4/31. (A) Relative cell viability of 3D4/31 macrophages after treatment with DB and/or PMA $(2 \mathrm{nM})$ for $24 \mathrm{~h}$. (B,C) Growth curve analysis of 3D4/31 macrophages; DB $(9 \mathrm{ng} / \mathrm{mL})$, and PMA (2 nM) replaced every $24 \mathrm{~h}$. Cell number (B) and phenotype (C) of 3D4/31 macophages $(\times 10$, scale bar $=100 \mu \mathrm{m})$. DB, Dudleya brittonii extract; PMA, phorbol 12-myristate 13-acetate. All data represent the mean \pm standard deviation $(n=$ $3) ;{ }^{*} p<0.05,{ }^{* *} p<0.01,{ }^{* * *} p<0.001$.

exert their functions, thus activating the antioxidant system even further to lower the ROS level. Therefore, we believe that the antioxidant effect of DB on 3D4/31 macrophages will have a positive effect on the function and lifespan of M2type AMs.

\section{Dudleya brittonii extract treatment induces lipid accumulation}

After demonstrating that DB effectively inhibits 3D4/31 macrophage cell death induced by exposure to PMA, we anticipated the possibility that $\mathrm{DB}$ may interfere with the activation of macrophages or cause metabolic problems in 3D4/31 macrophages. Since activated M2-like macrophages focus on lipid metabolism, we investigated the effect of DB on lipid metabolism of 3D4/31 macrophages by investigating for lipid production and its associated mechanism. To accomplish this, we measured lipid production by oil red $\mathrm{O}$ staining after 24 hours of DB and/or PMA treatment. DB (7.91\% \pm $4.29 \%)$ and PMA $(14.07 \% \pm 1.72 \%)$ treatments increased the lipid production of 3D4/31 macrophages, and the highest increase $(25.92 \% \pm 6.16 \%)$ was confirmed after co-treatment of PMA and DB (Figure 3A, 3B; $\mathrm{p}<0.05$ ). This confirms that DB has a positive effect on the lipid metabolism of 3D4/31 macrophages.

In addition, we confirmed alterations of FAS pathway genes expression in 3D4/31 macrophages by qPCR, after treatment with $\mathrm{DB}$ and/or PMA for $12 \mathrm{~h}$. PMA treatment upregulated the mRNA expression of ACACA and FASN, which are both crucial in the FAS pathway, and expression levels of ACACA and FASN were greater under conditions of co-treatment as 
A

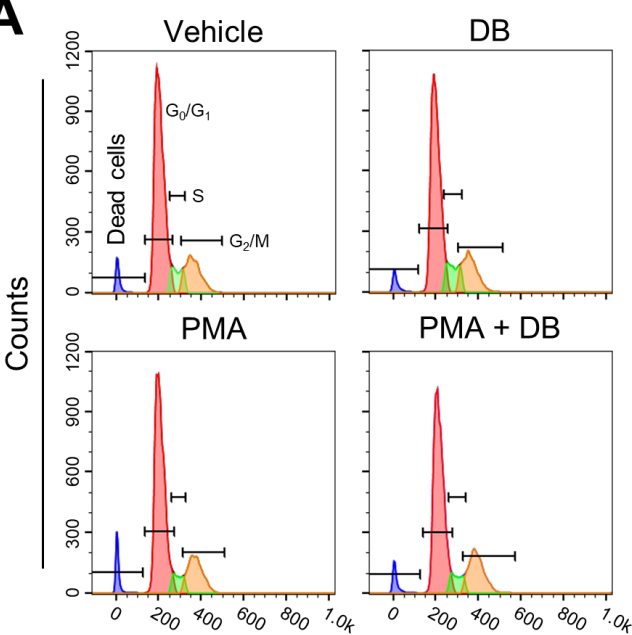

DNA contents
B

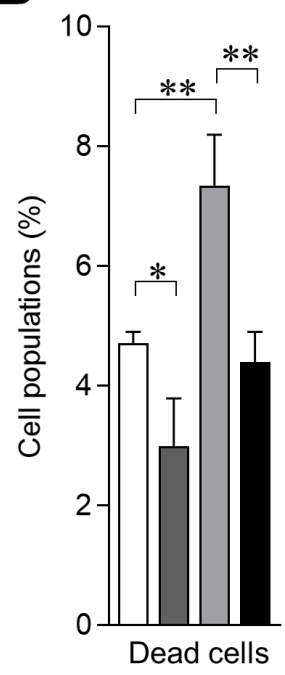

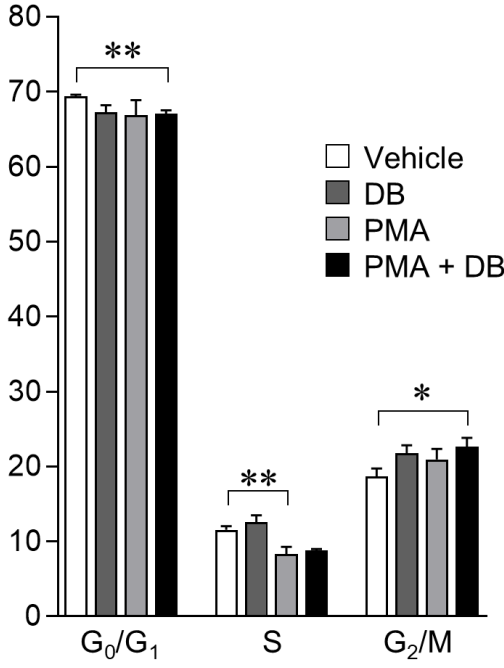
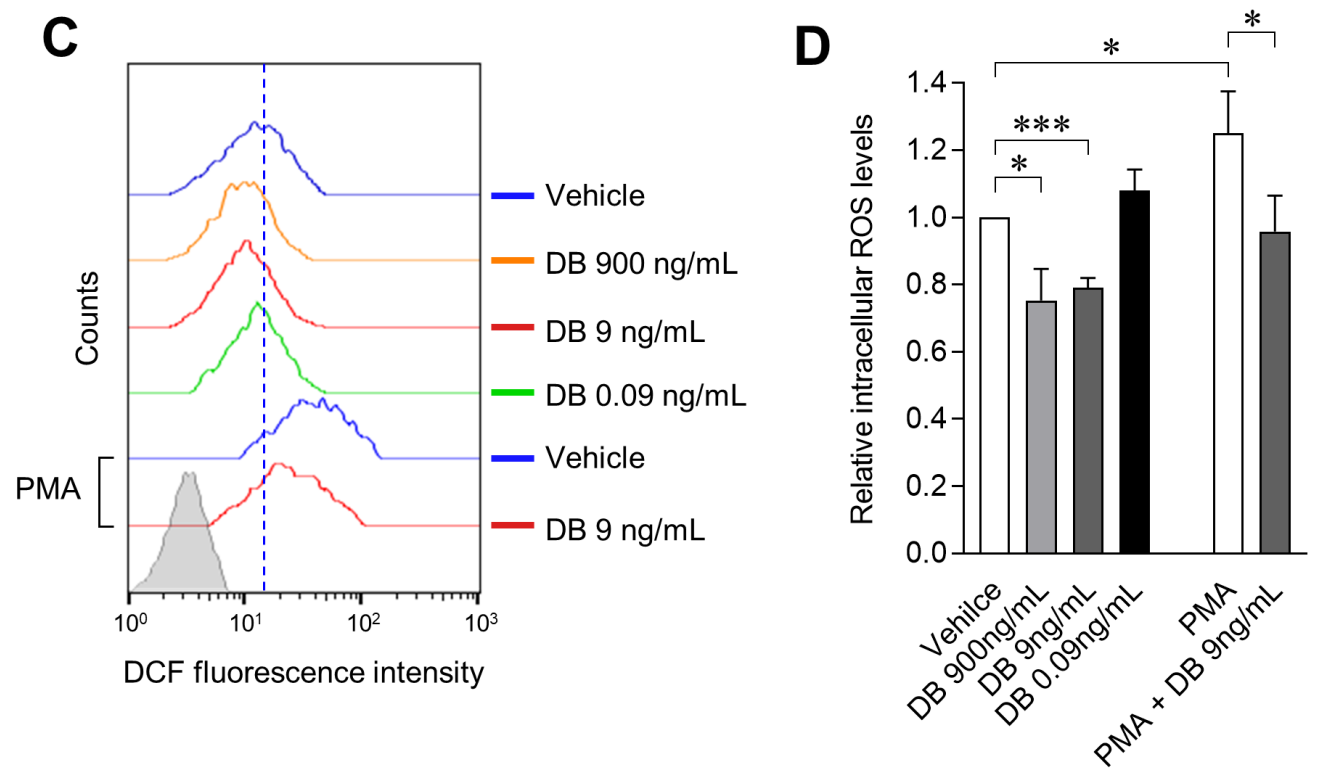

Figure 2. Anticytotoxic and antioxidant effects of DB on PMA treated 3D4/31 macrophages. (A, B) Cell cycle analysis of 3D4/31 macrophages: DB (9 ng/mL) and/or PMA (2 $\mathrm{nM}$ ) was treated for $24 \mathrm{~h}$. Based on flow cytometric profiles (A), dead cell populations (B) are increased by PMA treatment and reduced after DB exposure. (C, D) Intracellular ROS levels of 3D4/31 macrophages, after exposure to DB or/and PMA (2 nM) for $12 \mathrm{~h}$. Representative flow cytometric profiles (C) and relative intracellular ROS levels (D), was measured via flow cytometry with DCF-DA. DB, Dudleya brittonii extract; PMA, phorbol 12-myristate 13-acetate; ROS, reactive oxygen species; DCF-DA, dichlorofluorescein diacetate. All data represent the mean \pm standard deviation $(n=3) ;{ }^{*} p<0.05,{ }^{* *} p<0.01,{ }^{* * *} p<0.001$.

compared to PMA single treatment. These results indicate that DB enhances the FAS of PMA-activated 3D4/31 macrophages by controlling the ACACA and FASN expression (Figure 3C; $\mathrm{p}<0.05)$. In addition, expression of SRC3 was increased by PMA and DB-PMA co-treatment, suggesting induction of M2-like differentiation of 3D4/31 macrophages, and normal functioning of the sensor mechanism to regulate lipogenesis [27].

Unlike M1 macrophages which mainly utilize glycolysis, M2-like macrophages use the lipid metabolism. However, there are few studies reporting the changes in lipid production of porcine AMs. In this study, we confirm that PMA and DB upregulate the FAS pathway of porcine AMs, and increase lipid accumulation. Therefore, our results indicate that PMA induced activation is accompanied by lipid accumulation of porcine lung macrophages. In addition, since FAS plays a key role in phagocytic differentiation in monocytes [28] (a macrophage precursor cell), it is expected that administration of DB does not adversely affect the anti-bacterial function even if lipid production is upregulated in porcine CMs. 

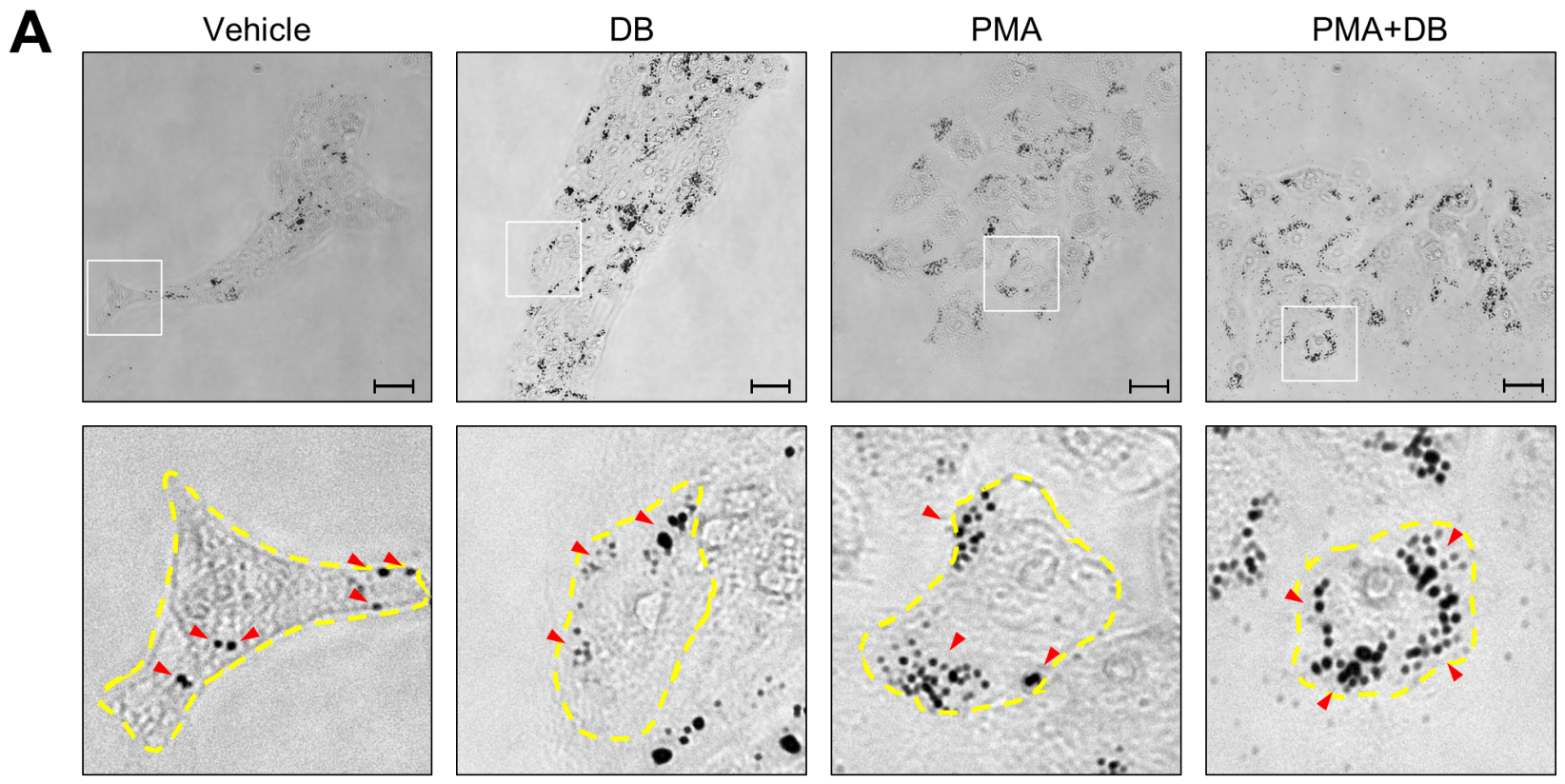

$\mathbf{B}$

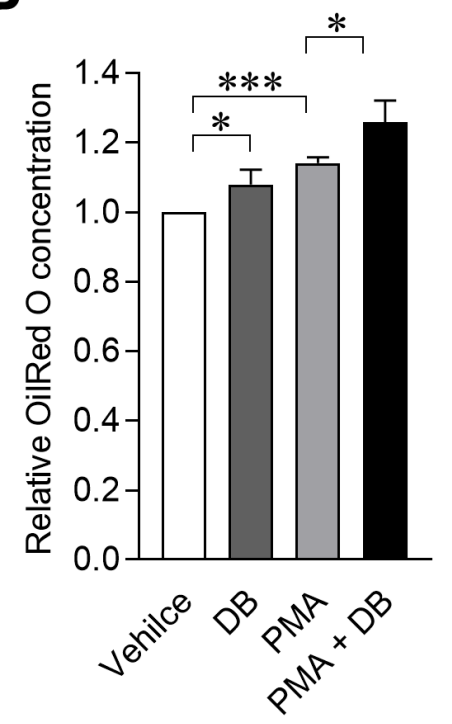

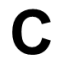

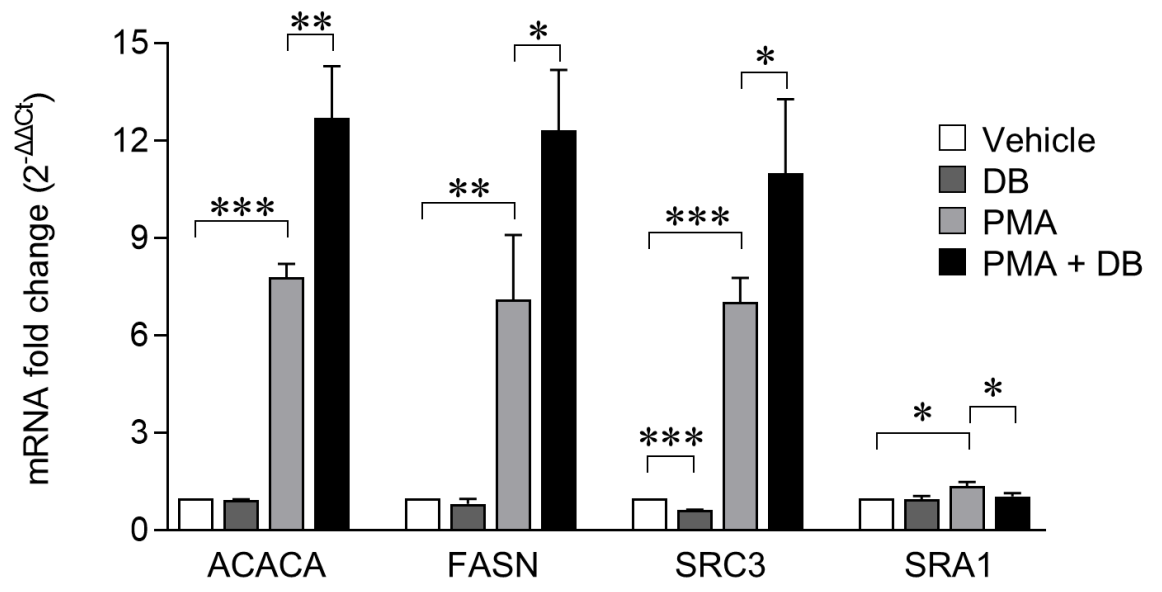

Figure 3. DB upregulates the fatty acid synthesis in 3D4/31 macrophages. (A) Oil red 0 staining of 3D4/31 macrophages after treatment with DB $(9 \mathrm{ng} / \mathrm{mL})$ and/or PMA (2 $\mathrm{nM})$ for $24 \mathrm{~h}(\times 20$, scale bar $=25 \mu \mathrm{m})$. Yellow line: single cell area, red arrow: oil red 0 stained area. (B) Relative oil red 0 concentration $(0 . D 492 \mathrm{~nm})$. Lipid synthesis and accumulation increases after exposure to DB and PMA. (C) qPCR analysis of fatty acid synthesis related genes. DB, Dudleya brittonii extract; PMA, phorbol 12-myristate 13-acetate; $q P C R$, quantitative polymerase chain reaction. All data represent the mean \pm standard deviation $(n=3) ;{ }^{*} p<0.05,{ }^{* *} p<0.01,{ }^{* * *} p<0.001$.

Dudleya brittonii extract treatment results in fatty acid oxidation biased metabolic change in 3D4/31

macrophages

Along with FAS, FAO plays a key role in the M2-like differentiation of AMs. We therefore performed a qPCR to verify the effect of $\mathrm{DB}$ and PMA on the 3 basic energy metabolisms (i.e., glycolysis, FAO, and TCA cycle) in 3D4/31 macrophages. For this assay, we measured the 27 gene expression levels in 3D4/31 macrophages after exposure to DB and/or PMA for
$12 \mathrm{~h}$. Treatment with DB alone resulted in decreased expression of glycolysis genes (glucose transporter 1 [GLUT1], hexokinase $[H K]$ 2, HK3, phosphofructokinase platelet $[P F K P]$, phosphofructokinase liver $[P F K L]$, pyruvate kinase liver and red blood cells $[P K L R]$, pyruvate kinase muscle $[P K M])$, and increased expression of FAO genes (long- chain acyl-CoA dehydrogenase $[A C A D L]$, acetyl-CoA acyltransferase 1A [ACAA1], acetyl-CoA acyltransferase 2 [ACAA2], peroxisome proliferator-activated receptor a $[P P A R A])$ and TCA 
cycle genes (dihydrolipoamide S-acetyltransferase [DLAT], citrate synthase [CTS], isocitrate dehydrogenase 3 alpha [IDH3], succinate thiokinase [SUCLG1], malate dehydrogenase [MDH] 1, $M D H 2$ ), as compared to the vehicle (Figure $4 \mathrm{~A}, 4 \mathrm{~B}$, and $4 \mathrm{C}$, respectively; $\mathrm{p}<0.05$ ). This result suggests that $\mathrm{DB}$ induces an M2-like metabolic change in 3D4/31 macrophages that are not yet activated. However, the expression levels of gly- colysis, FAO, and TCA cycle genes were not significantly changed when 3D4/31 macrophages were activated by PMA treatment for $12 \mathrm{~h}$; only a small number of gene expressions (ACACA, CTS) were increased, as compared to the vehicle. Finally, co-treatment with PMA and DB resulted in enhanced reduction in the expression of glycolysis genes (GLUT1, HK2, HK3, PFKP, PFKL, PKLR, PKM), and increase in the expres-

A

B
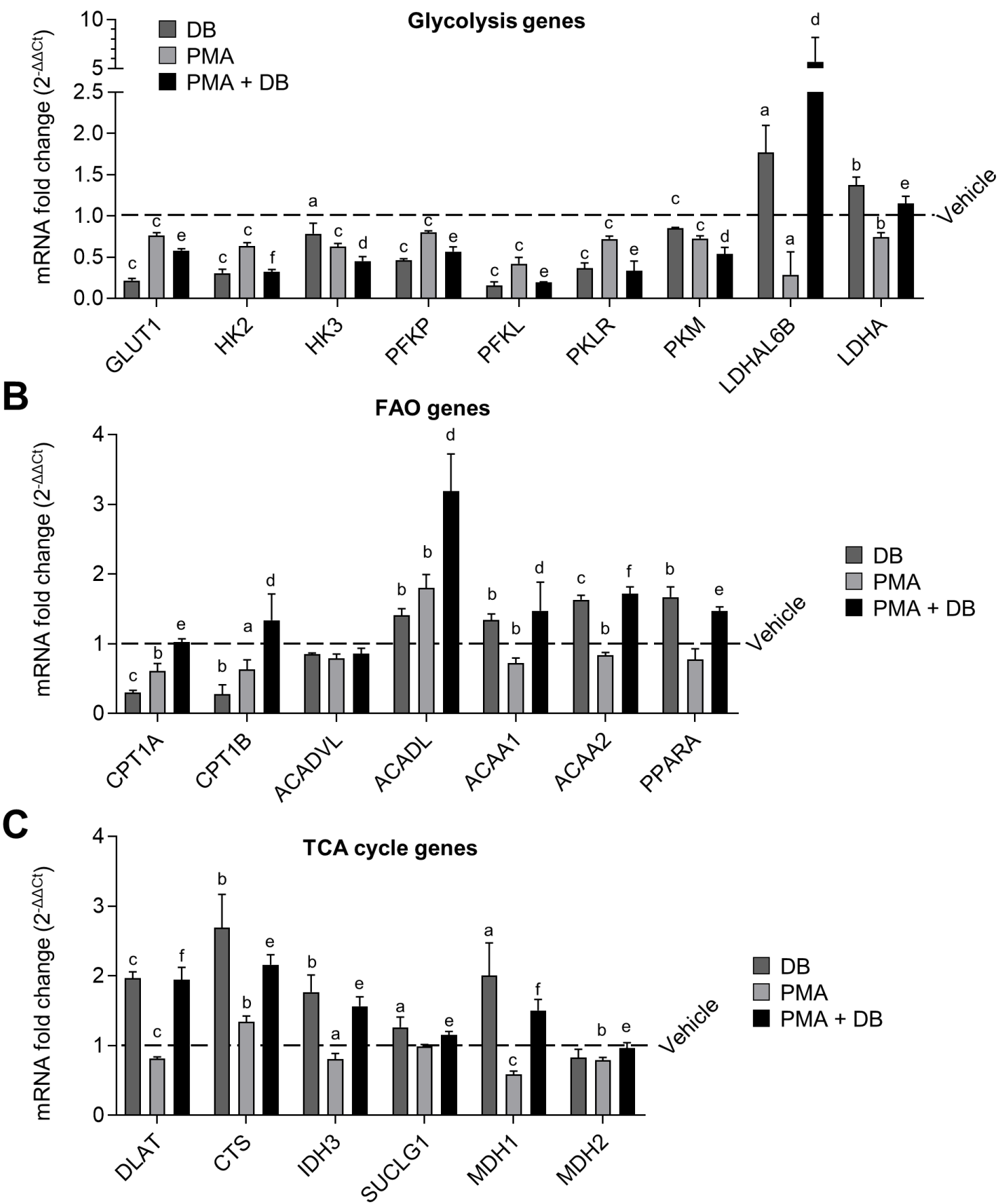

Figure 4. M2-like metabolic reprograming by DB in 3D4/31 macrophages. ( $A, B, C)$ Fold change of mRNA related with glycolysis (A), fatty acid oxidation (B), and TCA cycle (C). qPCR analysis was performed after treatment with DB ( $9 \mathrm{ng} / \mathrm{mL})$ and/or PMA (2 nM) for $12 \mathrm{~h}$. DB extract promotes the M2-like metabolic reprogramming, decreases glycolysis, and increases FAO and TCA cycle. DB, Dudleya brittonii extract; TCA, tricarboxylic acid; PMA, phorbol 12-myristate 13-acetate; qPCR, quantitative polymerase chain reaction. All data represent the mean \pm standard deviation $(n=3)$; vs vehicle ${ }^{\mathrm{a}} p<0.05,{ }^{b} p<0.01,{ }^{\mathrm{C}} p<0.001$; vs $\mathrm{PMA}{ }^{\mathrm{d}} p<0.05,{ }^{\mathrm{e}} p<0.01,{ }^{\mathrm{f}} p<0.001$. 
sion of FAO (carnitine palmitoyltransferase 1a [CPT1A], CPT1B, ACADL, ACAA1, ACAA2, PPARA) and TCA cycle genes (DLAT, CTS, IDH3, SUCLG1, MDH1, MDH2), compared to PMA treatment alone.

In addition, the specific expression of anaerobic glycolysis genes (L-lactate dehydrogenase A-like 6B-like isoform [LDHAL6B], lactate dehydrogenase A $[L D H A]$ ) was confirmed to be increased after DB treatment alone or co-treatment with PMA. Also, an unusual expression of the anaerobic glycolysis genes ( $L D H A L 6 B, L D H A$ ) was observed in the case of DB single-treatment or co-treatment with PMA. M2like macrophages such as AMs use FAO as an important factor for differentiation [29]; thus, up-regulation of FAO by $\mathrm{DB}$ is expected to positively affect the M2-like differentiation without inhibiting activity and function of 3D4/31 macrophages.

\section{DISCUSSION}

In this study, we identified DB to exert an M2-like activation and cell survival rate enhancement effect for AMs in vitro.
Our results demonstrate that DB inhibits the PMA induced ROS production, cell death, anti-proliferation, and anti-viability effect, and upregulates the FAS, FAO, and TCA cycle pathways in 3D4/31 macrophages (Figure 5). Furthermore, exposure to DB upregulates the specific energy metabolism of FAO and TCA cycle, but not glycolysis. We found further evidence in the role of DB in lipid metabolism during differentiation of porcine AMs. Taken together, our results indicate that DB has the potential to enhance the porcine alveolar immunity to prevent respiratory infectious diseases.

CMs derived from bone marrow possess a 2 to 4 day retention time in the blood [30], and although AMs have a much longer lifetime, their turnover rate is only $40 \%$ per year. Therefore, it is very important to keep the existing alveolar macrophage population healthy. Activation of AMs due to respiratory infections is a major threat to the AM population that exists from the time of birth; thus, the survival rate-enhancing efficacy of $\mathrm{DB}$ on activated AMs can be helpful for maintaining the respiratory health. AMs function even under normal conditions, even if there is no infection. Most functions of CMs are focused on the phagocytosis of the

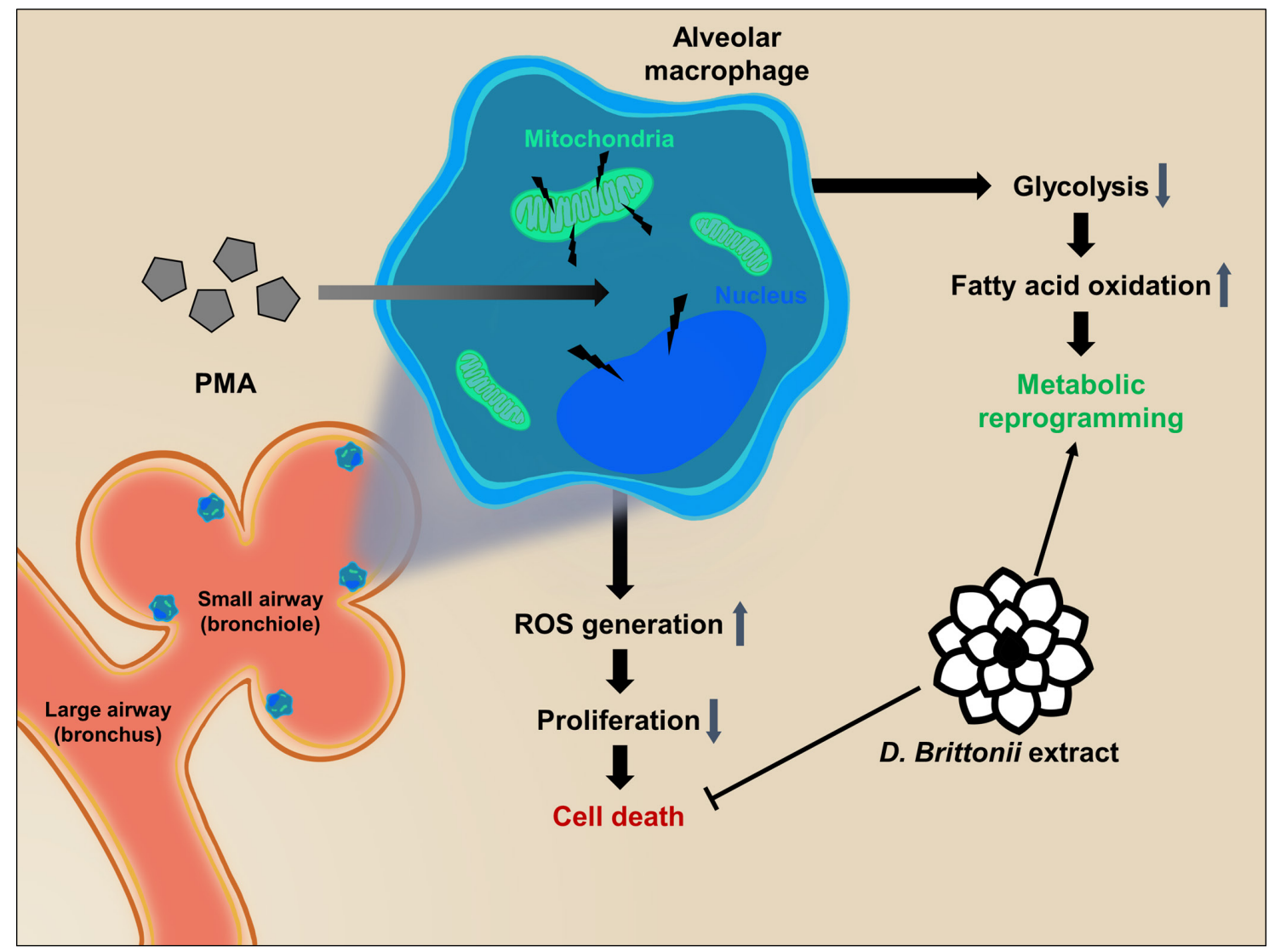

Figure 5. DB upregulates the cell survival rate of 3D4/31 by antioxidant and fatty acid oxidation. Alveolar macrophages are located in bronchioles to protect the lung against infections. DB downregulates the glycolysis gene expression and upregulates the fatty acid oxidation and TCA cycle genes, thereby promoting the M2-like metabolic reprogramming of 3D4/31 macrophages. Moreover, DB reduces macrophage activation (PMA induced) induced ROS generation and cell death in vitro. Taken together, DB upregulates the survival rate of 3D4/31 macrophages. DB, Dudleya brittonii extract; TCA, tricarboxylic acid; PMA, phorbol 12-myristate 13-acetate; ROS, reactive oxygen species. 
pathogen, but tissue-specific macrophages play an important role in maintaining homeostasis by removing apoptotic cells or cellular debris. Especially, AMs are closely related to inflammation and various diseases of the respiratory system. Pneumonia, Pasteurella multocida, foot-and-mouth disease virus, porcine respiratory coronavirus, porcine reproductive and respiratory syndrome virus, Streptococcus suis, Haemophilus parasuis, etc., cause great losses in the entire farmhouse because of their high infectivity [31-33]. Therefore, the survival rate enhancement effect of DB on AMs may help to prevent respiratory infectious diseases by improving the respiratory immunity of livestock. In addition, pneumonia is mainly caused by infections such as bacteria and viruses. In pigs, pneumonia easily caused by Mycoplasma pneumonia, Pasteurella multocida, etc., or complications [34]. Pig pneumonia has a high infection rate and spreads easily on the farms, which inhibit the porcine survival and growth, so proper treatment and prevention are important, but there is no proper treatment or prevention other than antibiotics. However, since the use of feed additive antibiotics is prohibited, a method of improving immunity to prevent pneumonia has been attempted. Since AMs play an important role in the control of pneumonia through anti-inflammatory and phagocytic actions, DB may be useful for the prevention of swine pneumonia $[35,36]$.

Growth hormones and feed additive antibiotics have been used indiscriminately in an age when there is no regulation on livestock production, to promote the growth of livestock and prevent diseases. However, their use has been banned in most countries due to environmental pollution and residue of animal products [37]. As a result, due to the precise correlation between steroid (or antibiotics) and infectious diseases, the repercussion has been an increase in the incidence of diseases in livestock. Hence, the demand for newer methods has increased and is newly developed to replace steroid (or antibiotics) [38]. Currently, steroids can be used for the recovery from bacterial infections or lung diseases, but they have no direct effect on the removal of infections and have been reported to only play a role in helping recovery $[39,40]$. In this study, we observed increased survival rate of AMs with SRC3 expression, which is a steroid sensor. It has previously been reported that steroid treatment prevents livestock diseases, and lung infections are improved by steroid sensor stimulation such as SRC3 of AMs.

This study confirms that DB upregulates the FAS, FAO, and TCA cycle genes, as well as SRC3 mRNA expression and cell survival rate in 3D4/31 AMs. However, the reason for the increase in LDHA and the decrease in WST-1 cell viability due to DB treatment is difficult to explain. These irregular results are expected to be induced by anaerobic glycolysis [41] or antioxidant effect. WST-1 assay is based on the activity of mitochondrial dehydrogenase (M-DHase) [42], M-DHase carries out energy production through oxidative phosphorylation on mitochondria, which is the largest part of intracellular ROS production [43]. Therefore, if the antioxidant activity of DB is accompanied by the inhibition of M-DHase activity, it is expected that similar results can be obtained as a decrease of viability with the decrease of ROS. In addition, further research in respiratory immunity using small animals such as a mouse needs to be performed in vivo, before practical application to livestock. Despite these limitations, this study confirms a new mechanism for the association of lipid metabolism and survival enhancement in the porcine AMs, and suggests that administering DB could potentially improve the survival rate of AMs. This study provides new insights and directions for further research in the immunity of the porcine respiratory system based on AMs and natural materials.

\section{CONFLICT OF INTEREST}

We certify that there is no conflict of interest with any financial organization regarding the material discussed in the manuscript.

\section{ACKNOWLEDGMENTS}

This research was supported by Agri-Bio Industry Technology Development Program (Project No. 316005-5), Ministry of Agriculture, Food and Rural Affairs (MAFRA), Republic of Korea. This work was also supported by a grant from the NextGeneration BioGreen 21 Program (Project No. PJ01323005), Rural Development Administration, Republic of Korea.

\section{REFERENCES}

1. Dittmar J, Janssen H, Kuske A, Kurtz J, Scharsack JP. Heat and immunity: an experimental heat wave alters immune functions in three-spined sticklebacks (Gasterosteus aculeatus). J Anim Ecol 2014;83:744-57. https://doi.org/10.1111/1365-2656.12175

2. Enting H, Boersma WJ, Cornelissen JB, Van Winden SC, Verstegen MW, Van Der Aar PJ. The effect of low-density broiler breeder diets on performance and immune status of their offspring. Poult Sci 2007;86:282-90. https://doi.org/10. 1093/ps/86.2.282

3. Hamad SH, Schauer JJ, Antkiewicz DS, Shafer MM, Kadhim AK. ROS production and gene expression in alveolar macrophages exposed to $\mathrm{PM}_{2.5}$ from Baghdad, Iraq: Seasonal trends and impact of chemical composition. Sci Total Environ 2016; 543:739-45. https://doi.org/10.1016/j.scitotenv.2015.11.065

4. Casewell M, Friis C, Marco E, McMullin P, Phillips I. The European ban on growth-promoting antibiotics and emerging consequences for human and animal health. J Antimicrob Chemother 2003;52:159-61. https://doi.org/10.1093/jac/ 
dkg313

5. Pabst R. The respiratory immune system of pigs. Vet Immunol Immunopathol 1996;54:191-5. https://doi.org/10.1016/S01652427(96)05700-5

6. Guilliams M, De Kleer I, Henri S, et al. Alveolar macrophages develop from fetal monocytes that differentiate into long-lived cells in the first week of life via GM-CSF. J Exp Med 2013;210: 1977-92. https://doi.org/10.1084/jem.20131199

7. Hussell T, Bell TJ. Alveolar macrophages: plasticity in a tissuespecific context. Nat Rev Immunol 2014;14:81-93. https:// doi.org/10.1038/nri3600

8. Landsman L, Jung S. Lung macrophages serve as obligatory intermediate between blood monocytes and alveolar macrophages. J Immunol 2007;179:3488-94. https://doi.org/10.4049/ jimmunol.179.6.3488

9. Nomura M, Liu J, Rovira II, et al. Fatty acid oxidation in macrophage polarization. Nat Immunol 2016;17:216-7. https://doi. org/10.1038/ni.3366

10. Menendez JA, Lupu R. Fatty acid synthase and the lipogenic phenotype in cancer pathogenesis. Nat Rev Cancer 2007;7: 763-77. https://doi.org/10.1038/nrc2222

11. Carracedo A, Cantley LC, Pandolfi PP. Cancer metabolism: fatty acid oxidation in the limelight. Nat Rev Cancer 2013;13: 227-32. https://doi.org/10.1038/nrc3483

12. Jin J, Wang T, Wang Y, et al. SRC3 expressed in BMSCs promotes growth and migration of multiple myeloma cells by regulating the expression of Cx43. Int J Oncol 2017;51:1694704. https://doi.org/10.3892/ijo.2017.4171

13. Wu M-Y, Fu J, Xu J, O'malley BW, Wu R-C. Steroid receptor coactivator 3 regulates autophagy in breast cancer cells through macrophage migration inhibitory factor. Cell Res 2012;22: 1003-21. https://doi.org/10.1038/cr.2012.44

14. York B, Reineke EL, Sagen JV, et al. Ablation of steroid receptor coactivator-3 resembles the human CACT metabolic myopathy. Cell Metab 2012;15:752-63. https://doi.org/10.1016/ j.cmet.2012.03.020

15. Chen Q, Chen T, Xu Y, et al. Steroid receptor coactivator 3 is required for clearing bacteria and repressing inflammatory response in Escherichia coli-induced septic peritonitis. J Immunol 2010;185:5444-52.

16. Savoia D. Plant-derived antimicrobial compounds: alternatives to antibiotics. Future Microbiol 2012;7:979-90.

17. Arakaki M, Christin P-A, Nyffeler R, et al. Contemporaneous and recent radiations of the world's major succulent plant lineages. Proc Natl Acad Sci USA 2011;108:8379-84. https:// doi.org/10.1073/pnas.1100628108

18. Park M-Y, Kwon H-J, Sung M-K. Evaluation of aloin and aloe-emodin as anti-inflammatory agents in aloe by using murine macrophages. Biosci Biotechnol Biochem 2009;73: 828-32. https://doi.org/10.1271/bbb.80714

19. Schepetkin IA, Xie G, Kirpotina LN, Klein RA, Jutila MA, Quinn MT. Macrophage immunomodulatory activity of poly- saccharides isolated from Opuntia polyacantha. Int Immunopharmacol 2008;8:1455-66. https://doi.org/10.1016/j.intimp. 2008.06.003

20. Mulroy TW. Spectral properties of heavily glaucous and nonglaucous leaves of a succulent rosette-plant. Oecologia 1979; 38:349-57. https://doi.org/10.1007/BF00345193

21. Kim S-J, Hwang E, Yi SS, et al. Sea buckthorn leaf extract inhibits glioma cell growth by reducing reactive oxygen species and promoting apoptosis. Appl Biochem Biotechnol 2017;182: 1663-74. https://doi.org/10.1007/s12010-017-2425-4

22. Kim H, Yi SS, Lee H-K, et al. Antiproliferative effect of vine stem extract from Spatholobus suberectus Dunn on rat C6 glioma cells through regulation of ROS, mitochondrial depolarization, and P21 protein expression. Nutr Cancer 2018;70:60519. https://doi.org/10.1080/01635581.2018.1460673

23. Keyel PA, Tkacheva OA, Larregina AT, Salter RD. Coordinate stimulation of macrophages by microparticles and TLR ligands induces foam cell formation. J Immunol 2012;189:4621-9. https://doi.org/10.4049/jimmunol.1200828

24.Livak KJ, Schmittgen TD. Analysis of relative gene expression data using real-time quantitative PCR and the 2(-Delta Delta C(T)) method. Methods 2001;25:402-8. https://doi.org/10. 1006/meth.2001.1262

25. Ferrante CJ, Leibovich SJ. Regulation of macrophage polarization and wound healing. Adv Wound Care 2012;1:10-6. https://doi.org/10.1089/wound.2011.0307

26. Fortes GB, Alves LS, de Oliveira R, et al. Heme induces programmed necrosis on macrophages through autocrine TNF and ROS production. Blood 2012;119:2368-75. https://doi. org/10.1182/blood-2011-08-375303

27.Li J, Liu Y-H, Ou S, Dai X-M, Wang J-P, Su Y-P. Steroid receptor coactivator-3 differentially regulates the inflammatory response in peritoneal macrophages. Mol Med Rep 2012;5: 1099-105. https://doi.org/10.3892/mmr.2012.750

28.Ecker J, Liebisch G, Englmaier M, Grandl M, Robenek H, Schmitz G. Induction of fatty acid synthesis is a key requirement for phagocytic differentiation of human monocytes. Proc Natl Acad Sci USA 2010;107:7817-22. https:/doi.org/10.1073/ pnas.0912059107

29. Mills EL, O'Neill LA. Reprogramming mitochondrial metabolism in macrophages as an anti-inflammatory signal. Eur J Immunol 2016;46:13-21. https://doi.org/10.1002/eji.201 445427

30.Patel AA, Zhang Y, Fullerton JN, et al. The fate and lifespan of human monocyte subsets in steady state and systemic inflammation. J Exp Med 2017;214:1913-23. https://doi.org/ 10.1084/jem.20170355

31. Brockmeier SL, Halbur PG, Thacker EL. Porcine respiratory disease complex. In: Brogden KA, Guthmiller JM, editors. Polymicrobial diseases. Washington, DC, USA: ASM Press; 2002.

32. Renukaradhya GJ, Alekseev K, Jung K, Fang Y, Saif LJ. Porcine 
reproductive and respiratory syndrome virus-induced immunosuppression exacerbates the inflammatory response to porcine respiratory coronavirus in pigs. Viral Immunol 2010; 23:457-66. https://doi.org/10.1089/vim.2010.0051

33. Opriessnig T, Giménez-Lirola L, Halbur P. Polymicrobial respiratory disease in pigs. Anim Health Res Rev 2011;12:133-48. https://doi.org/10.1017/S1466252311000120

34. Amass S, Clark L, Bowersock T, Murphy D, Knox K, Albregts S. Interaction of Mycoplasma hyopneumoniae and Pasteurella multocida infections in swine. J Am Vet Med Assoc 1994;204: 102-7.

35. Knapp S, Leemans JC, Florquin S, et al. Alveolar macrophages have a protective antiinflammatory role during murine pneumococcal pneumonia. Am J Respir Crit Care Med 2003;167: 171-9. https://doi.org/10.1164/rccm.200207-698OC

36. Aberdein J, Cole J, Bewley M, Marriott H, Dockrell D. Alveolar macrophages in pulmonary host defence-the unrecognized role of apoptosis as a mechanism of intracellular bacterial killing. Clin Exp Immunol 2013;174:193-202. https://doi. org/10.1111/cei.12170

37.Sundlof SF. Drug and chemical residues in livestock. Vet Clin North Am Food Anim Pract 1989;5:411-49. https://doi.org/ 10.1016/S0749-0720(15)30984-1

38. Li J. Current status and prospects for in-feed antibiotics in the different stages of pork production - a review. Asian-Australas J Anim Sci 2017;30:1667-73. https://doi.org/10.5713/ajas.17. 0418

39.Spector SL, Katz FH, Farr RS. Troleandomycin: effectiveness in steroid-dependent asthma and bronchitis. J Allergy Clin Immunol 1974;54:367-79. https://doi.org/10.1016/0091-6749 (74)90027-X

40. Martens A, Boada M, Vanaudenaerde BM, et al. Steroids can reduce warm ischemic reperfusion injury in a porcine donation after circulatory death model with ex vivo lung perfusion evaluation. Transpl Int 2016;29:1237-46. https://doi.org/10. 1111/tri.12823

41.Fantin VR, St-Pierre J, Leder P. Attenuation of LDH-A expression uncovers a link between glycolysis, mitochondrial physiology, and tumor maintenance. Cancer Cell 2006;9:425-34. https://doi.org/10.1016/j.ccr.2006.04.023

42. Toimela T, Tähti H. Mitochondrial viability and apoptosis induced by aluminum, mercuric mercury and methylmercury in cell lines of neural origin. Arch Toxicol 2004;78:565-74. https://doi.org/10.1007/s00204-004-0575-y

43. Adam-Vizi V, Tretter L. The role of mitochondrial dehydrogenases in the generation of oxidative stress. Neurochem Int 2013;62:757-63. https://doi.org/10.1016/j.neuint.2013.01.012 